\title{
Intérêt de l'échographie des glandes salivaires dans le diagnostic du syndrome de Gougerot-Sjögren
}

\author{
Beuzit $\mathrm{S}^{1}$, Boisramé Gastrin $\mathrm{S}^{1}$, Pers $\mathrm{JO}^{1}$, Jousse Joulin $\mathrm{S}^{2}$ \\ ${ }^{1}$ UFR d'Odontologie, CHRU, Brest, France \\ ${ }^{2}$ UFR de Médecine, CHRU, Brest, France \\ beuzit.sebastien@gmail.com
}

Le syndrome de Gougerot-Sjögren (SGS) est une maladie auto-immune affectant les glandes exocrines, notamment les glandes salivaires et lacrymales. Les principaux symptômes sont la xérophtalmie et la xérostomie. La prévalence de cette atteinte varie de 0.5 à $3 \%$ selon les études et touche principalement la femme (ratio de 9/1).

Les critères actuels de diagnostic de ce syndrome ont été définis par le groupe américanoeuropéen d'experts en 2002 et reposent sur 6 items : les symptômes oculaires et oraux, la mesure des flux lacrymaux et salivaires, la sialographie ou la scintigraphie des glandes salivaires, l'existence d'un infiltrat lymphocytaire dans les glandes salivaires accessoires et la présence d'auto-anticorps (Vitali et al. 2002).

Une consultation pluridisciplinaire dédiée au SGS existe au CHRU de Brest depuis 1990 et le bilan comporte depuis 2004 un examen dermatologique, ophtalmique, odonto-stomatologique, psychiatrique, rhumatologique et échographique. Il a été montré que des altérations échographiques des glandes salivaires sont retrouvées de façon fréquente, bien avant que les anomalies biologiques ne soient décelées.

L'échographie des glandes salivaires n'est pour l'instant pas reconnue comme critère officiel de diagnostic de la maladie. Son utilisation en pratique clinique est pourtant de plus en plus d'actualité, et de nombreux auteurs (Vera et al. 2009, Wernicke et al. 2009) ont tenté de démontrer son utilité et sa grande spécificité pour le SGS. Les avantages de cet examen sont sa simplicité de mise en œuvre, son caractère non-invasif et son faible coût. Certains auteurs (De Vita et al. 1992, Makula et al. 1996) estiment que cet examen ultrasonographique pourrait être réalisé en cas de suspicion de SGS, et qu'il permettrait de justifier d'autres examens plus invasifs comme la biopsie. De plus, l'échographie Doppler pulsé des glandes salivaires est certainement l'outil le plus intéressant pour le suivi des patients, et l'évaluation de la réponse aux traitements (Jousse Joulin et al. 2007).

Le but de cette présentation est d'illustrer, à travers différents cas cliniques, la place de l'échographie dans le diagnostic et le suivi du SGS, en s'appuyant sur les données de la littérature. 